\title{
At-wavelength Optical Metrology Development at the ALS
}

\author{
Sheng Yuan ${ }^{* 1}$, Kenneth A. Goldberg ${ }^{2}$, Valeriy V. Yashchuk ${ }^{1}$, Richard Celestre ${ }^{1}$, Iacopo Mochi ${ }^{2}$, \\ James Macdougall ${ }^{2}$, Gregory Y. Morrison ${ }^{3}$, Brian V. Smith ${ }^{3}$, Edward E. Domning, \\ Wayne R. McKinney ${ }^{1}$, Tony Warwick ${ }^{1}$ \\ ${ }^{1}$ Advanced Light Source, Lawrence Berkeley National Laboratory, Berkeley, CA 94720, USA \\ ${ }^{2}$ Center for X-Ray Optics, Lawrence Berkeley National Laboratory, Berkeley, CA 94720, USA \\ ${ }^{3}$ Engineering Division, Lawrence Berkeley National Laboratory, Berkeley, CA 94720, USA
}

\begin{abstract}
Nano-focusing and brightness preservation for ever brighter synchrotron radiation and free electron laser beamlines require surface slope tolerances of x-ray optics on the order of 100 nrad. While the accuracy of fabrication and ex situ metrology of x-ray mirrors has improved over time, beamline in situ performance of the optics is often limited by application specific factors such as x-ray beam heat loading, temperature drift, alignment, vibration, etc. In the present work, we discuss the recent results from the Advanced Light Source developing high accuracy, in situ, at-wavelength wavefront measurement techniques to surpass 100-nrad accuracy surface slope measurements with reflecting x-ray optics. The techniques will ultimately allow closed-loop feedback systems to be implemented for x-ray nano-focusing. In addition, we present a dedicated metrology beamline endstation, applicable to a wide range of in situ metrology and test experiments. The design and performance of a bendable Kirkpatrick-Baez (KB) mirror with active temperature stabilization will also be presented. The mirror is currently used to study, refine, and optimize in situ mirror alignment, bending and metrology methods essential for nano-focusing application.
\end{abstract}

Keywords: at-wavelength, optical metrology, nano-focusing, mirror alignment, slope measurement, interferometer, $\mathrm{x}$-ray optics, synchrotron radiation

\section{INTRODUCTION}

Nano-focusing and brightness preservation for ever-brighter synchrotron radiation and free electron laser beamlines require surface slope tolerances of reflective x-ray optics on the order of $100 \mathrm{nrad}$. While the accuracy of fabrication and ex situ metrology of x-ray mirrors has improved over time [1-6], beamline in situ, at-wavelength performance of the optics is often limited by application specific factors such as x-ray beam alignment, mechanical and temperature drift, vibration, etc. [7-11].

At the Advanced Light Source (ALS), we are developing broadly applicable, high-accuracy, in situ, at-wavelength wavefront measurement techniques to surpass 100-nrad slope measurement accuracy for Kirkpatrick-Baez (KB) mirrors [6]. The at-wavelength methodology relies on a series of tests with increasing accuracy and sensitivity [12]. For the purposes of development, these soft X-ray tests are performed on a single, bendable KB mirror during mirror alignment and optimization, thus enabling the inter-comparison of the various techniques. At this time, we are concentrating on the non-interferometric techniques, whose modest hardware requirements and straightforward analysis lends itself well to a wide variety of beamline configurations. Future studies will include the analysis of interferometric tests.

Combining multiple techniques with high-precision tuning methods enables us to perform in situ alignment and setting of bendable x-ray optics adequate for sub-100-nrad slope-error focusing.

Two of the most significant factors limiting nano-focusing in practice are vibration isolation and temperature stability. We have created a dedicated test chamber to mitigate these significant issues. The chamber configuration also enables multiple tests to be performed using shared hardware, and nanofabricated components.

\footnotetext{
*shyuan1977@gmail.com; phone +1-510-486-6729
} 
In many cases, reflective soft and hard x-ray optical systems are achromatic-grazing-incidence KB mirror systems typically fall into this category. Where this property is true, system optimization performed at one wavelength ensures optimization at other relevant wavelengths. Our tests are performed at soft x-ray wavelengths near $1 \mathrm{~nm}(\sim 1 \mathrm{keV})$, where the creation of nano-fabricated diffractive optical structures with high opacity is far simpler than for hard x-rays, and where efficient reflection at larger angles of incidence is possible.

Here we report on the current status of the at-wavelength metrology development at the ALS. We describe an original method for optimal alignment of the mirror in situ. Especially, we report that $\sim 120 \mathrm{~nm}$ FWHM focal size was achieved at 1-nm soft x-ray wavelength using a 100-mm-long temperature-stabilized KB mirror.

\section{ARRANGEMENT OF THE METROLOGY BEAMLINE ENDSTATION}

Metrology beamline 5.3.1 at the ALS has a versatile design with a broad energy range from $30 \mathrm{eV}$ to $12 \mathrm{keV}$, and a long optical bench formed with two connected optical tables. The beamline delivers light to the metrology test chamber.

Figure 1 shows the beamline layout. X-rays from an ALS bend magnet, are focused by a 1:1 toroidal mirror, M1, and pass through a monochromator, comprised of a pair of $\mathrm{W} / \mathrm{B}_{4} \mathrm{C}$ multilayer mirrors with 4-nm period. The monochromator is calibrated using the 4997-eV absorption edge of a 6-micron thick titanium foil.

The M1 toroidal mirror focuses the incident $\mathrm{x}$-rays 12-m downstream. The focal spot has a measured FWHM size of $150 \mu \mathrm{m}$ (vertical) $\times 300 \mu \mathrm{m}$ (horizontal). This focus is formed inside the 2-m-long vacuum chamber of the dedicated experimental endstation. The focused light passes through a circular, $200-\mu \mathrm{m}$-diameter, tungsten aperture intended to block stray light from reaching the mirror. The focus of the beamline coincides with the object plane of the test mirror.

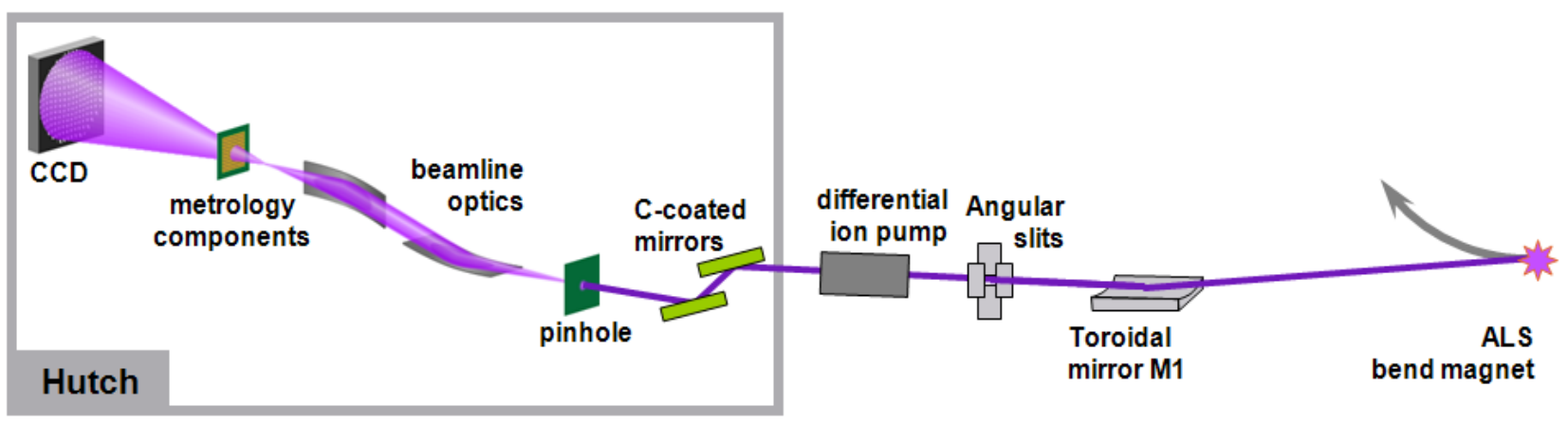

Figure 1. Arrangement of metrology beamline 5.3.1 at the ALS.

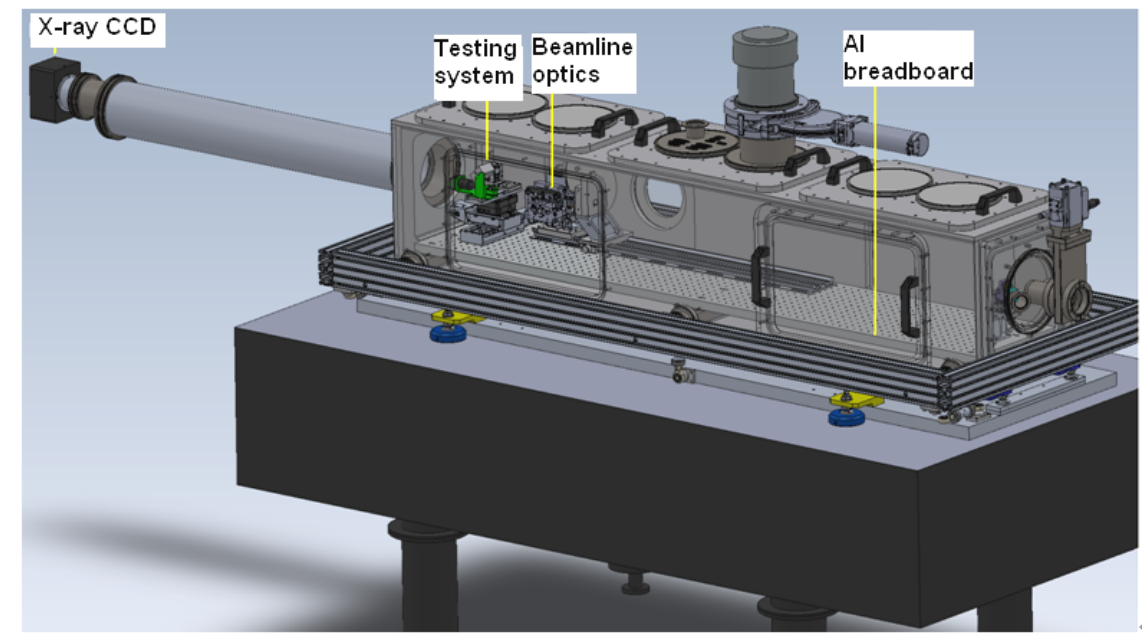

Figure 2. The 2-m-long vacuum chamber design. To achieve a high degree of stability and vibration isolation, the interior optical components, including the $\mathrm{KB}$ mirror assembly, are attached to a single breadboard, rigidly connected to the optical table through flexible bellows, and decoupled from chamber and environmental vibrations. The x-ray CCD camera extends $1.6 \mathrm{~m}$ beyond the focal plane. 
Figure 2 shows the 2-m-long vacuum chamber design, sitting on an optical table. Since mechanical vibration can degrade focusing performance, vibration isolation was a primary consideration in the endstation design. The interior optical breadboard, which supports the KB test mirror assembly and the optical components in both conjugate planes, is rigidly supported by the main optical table through six flexible bellows connections, and is thereby decoupled from the vibrations of the vacuum chamber.

\section{A BENDABLE TEST MIRROR WITH ACTIVE TEMPERATURE STABILIZATION}

The experiments described here were conducted with a single, bendable, KB test mirror prepared at the ALS Optical Metrology Laboratory (OML); the mirror slope profile has been reported previously [6]. Table 1 presents the mirror's optical specifications as used. The temperature-stabilized mirror-bender design in the present work is closely related to the design of KB mirrors fabricated for ALS beamline 12.3.2 [13].

Table 1. Specifications of the KB test mirror. The mirror is a flat, 4-mm-thick silicon substrate, with 102-mm length, and an 80-mm clear aperture. The mirror is side-profiled to generate an elliptical shape when optimally bent.

*The RMS slope error, measured with visible-light long-trace profilometry is shown in Fig. 3.

\begin{tabular}{l|l|l|l|l|l}
$\begin{array}{l}\text { mirror center radius } \\
\text { of curvature }\end{array}$ & $\begin{array}{l}\text { object } \\
\text { distance }\end{array}$ & $\begin{array}{l}\text { image } \\
\text { distance }\end{array}$ & $\begin{array}{l}\text { central ray } \\
\text { grazing angle }\end{array}$ & $\begin{array}{l}\text { numerical } \\
\text { aperture }\end{array}$ & $\begin{array}{l}\text { measured RMS } \\
\text { slope error* }\end{array}$ \\
\hline $27.91 \mathrm{~m}$ & $1600 \mathrm{~mm}$ & $120 \mathrm{~mm}$ & $8.0 \mathrm{mrad}$ & $2.67 \mathrm{mrad}$ & $0.4 \mu \mathrm{rad}$
\end{tabular}

Figure 3 shows the mirror's optimized, measured slope error prior to beamline installation. Measurements and bending optimization were performed with the upgraded ALS long trace profiler (LTP-II) at the OML, following an scanning strategy described in Ref. 6 . The optimization procedure removes defocus and coma aberrations $\left(2^{\text {nd }}\right.$ and $3^{\text {rd }}$ order shape errors) at the design conjugate distances. The rigid-body optimization of mirror displacement and tilt, however, must be performed in situ. Following alignment, the RMS residual slope error was $0.4 \mu \mathrm{rad}$. Ignoring diffraction, the expected geometrical RMS spot size can be estimated as $96 \mathrm{~nm}(2 \times$ RMS residual slope error $\times 120 \mathrm{~mm}$ focal length). By design, the upstream edge of the mirror's clear aperture is twice as far from the focal plane $(160 \mathrm{~mm})$ as the downstream edge $(80 \mathrm{~mm})$. Therefore, slope errors on the upstream side will contribute geometrical ray errors that are twice as large as those on the downstream side. Errors on the upstream edge are more significant for nano-focusing thus in situ mirror fine tuning is necessary.

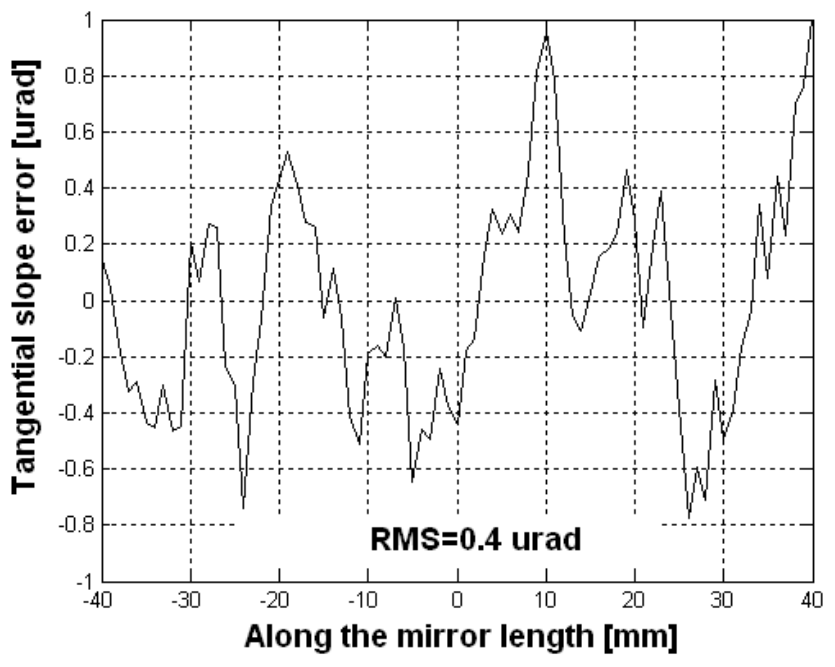

Figure 3. The residual error of the mirror tangential slope after subtraction of the ideal elliptical shape, measured in the sagittal center of the mirror and averaged over four consecutive measurements. The RMS residual slope error is $0.4 \mu \mathrm{rad}$. Ignoring diffraction, the expected RMS geometrical spot size expected from this mirror slope error is $\sim 96 \mathrm{~nm}$.

Figure 3 shows that the low-spatial-frequency slope aberrations have been removed to within the measurement uncertainty. However, at the beamline, real performance is often limited by alignment uncertainty (i.e. focal distance, displacement, pitch, roll, and yaw), mechanical and temperature drift, vibration, etc. [7-11]. The mirror bender assembly has five adjustments that can be controlled externally: two bending couples, pitch, roll and vertical-translation. Prior to 
installation, using a ZYGO GPI ${ }^{\mathrm{TM}}$, we carefully aligned the mirror roll and yaw angles to make the mirror surface parallel to the base plane of the mirror mounting bracket and the mirror's long-axis parallel to the beam propagation direction.

Thermal effects arising from ambient temperature fluctuations are among the most troubling environmental factors [11]. In principle, the bent mirror shape is highly sensitive to temperature variation because the aluminum bender assembly and the silicon mirror substrate are made from different materials with significantly different thermal expansion coefficients (approximately 23 and $3 \mathrm{ppm} /{ }^{\circ} \mathrm{C}$, respectively). As the ambient temperature varies at the beamline, temperature changes can affect the bending forces applied to the mirror, altering the shape and degrading the mirror performance [11].

In order to mitigate the temperature sensitivity, a Peltier temperature-stabilization system is attached to the KB mirror bender assembly (as shown in Fig. 4). Molybdenum glue-blocks are attached to the ends of the mirror to improve the thermal conductivity between the mirror and the bending mechanism. Using this system, we have previously demonstrated that temperature stabilization of the mirror-bender assembly stabilizes the mirror shape under several degrees of environmental temperature change [11].

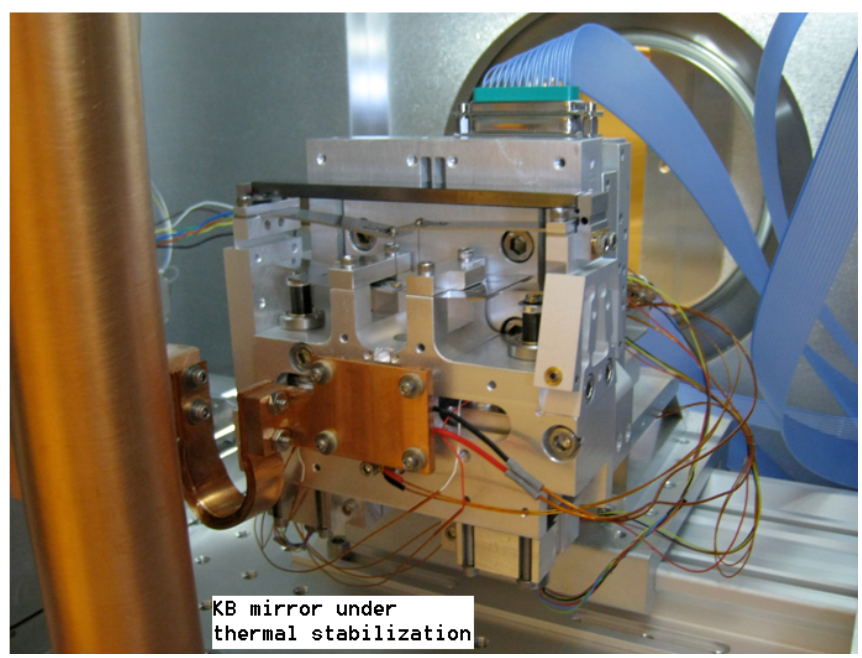

Figure 4. Upward-facing KB mirror held in the mirrorbender assembly with a Peltier thermal stabilization unit attached. The mirror bender has five externally controlled alignment adjustments: two bending couples, pitch, roll and vertical-translation. This photo was taken following installation in the test chamber.

\section{AT-WAVELENGTH TESTING METHODOLOGY}

Figure 5 illustrates a series of at-wavelength tests to be performed repeatedly during in situ alignment and tuning as the system approaches an optimized condition. Two of these, the scanning-slit test and the knife-edge test, were used to characterize and optimize the alignment of the single KB mirror used in these tests for demonstration purposes. This onedimensional focusing exercise enables us to isolate and study the performance of the various testing methods without the complication of two mirror reflections, yet the methods we describe can easily be extended to two-dimensional focusing.

All of the tests rely on well-conditioned illumination from a single point in the object plane of the test mirror. ALS beamline 5.3.1 provides a monochromatic beam $(E / \Delta E=500)$ focused to a $150 \mu \mathrm{m}$ (vertical) $\times 300 \mu \mathrm{m}$ (horizontal) spot. To generate a cylindrical reference beam for one-dimensional focusing tests, we place a nano-fabricated entrance-slit spatial filter in the object plane of the KB. An array of $200-\mu \mathrm{m}$-long slits with widths between 0.66 and $6.00 \mu \mathrm{m}$ was created by electron-beam lithography to enable optimal size to be selected experimentally. The structures have a $1-\mu \mathrm{m}-$ thick gold absorber layer on a 100 -nm silicon-nitride membrane.

\subsection{Scanning-slit and knife-edge tests}

The simplest and most widely used at-wavelength technique for $\mathrm{x}$-ray mirror alignment and tuning is the scanning- slit test $[14,15]$, which is essentially a temporal version of the well-known Hartmann test [16]. A movable slit placed before the mirror (Fig. 5a) restricts the illuminated portion of the mirror in steps and the lateral position of the line-focus is measured as a function of the beam position along the mirror's clear aperture (controlled by the slit position). In optimal alignment, the lateral line-focus in the focal plane should be stationary, indicating that all portions of the mirror surface 
are focused to the same position. The motion of the focused line reveals local slope variations along the mirror surface. Here by averaging along the line focus to calculate the meridional position, we restrict our attention to one dimension of focusing. However, sagittal information is available both by studying points along the line focus, which can be interpreted separately, or by performing a two-dimensional slit scan, isolating restricted positions along the mirror surface point by point. Owing to the mirror's elliptical shape and oblique angle of incidence, there is a non-linear relationship between the scanning-slit and the beam position on the mirror surface (Fig. 5a). The mirror alignment optimization we describe can be performed accurately, without regard to this non-linear relationship, yet we note that the accurate interpretation of the results as wavefront slope measurements requires a simple coordinate transformation.

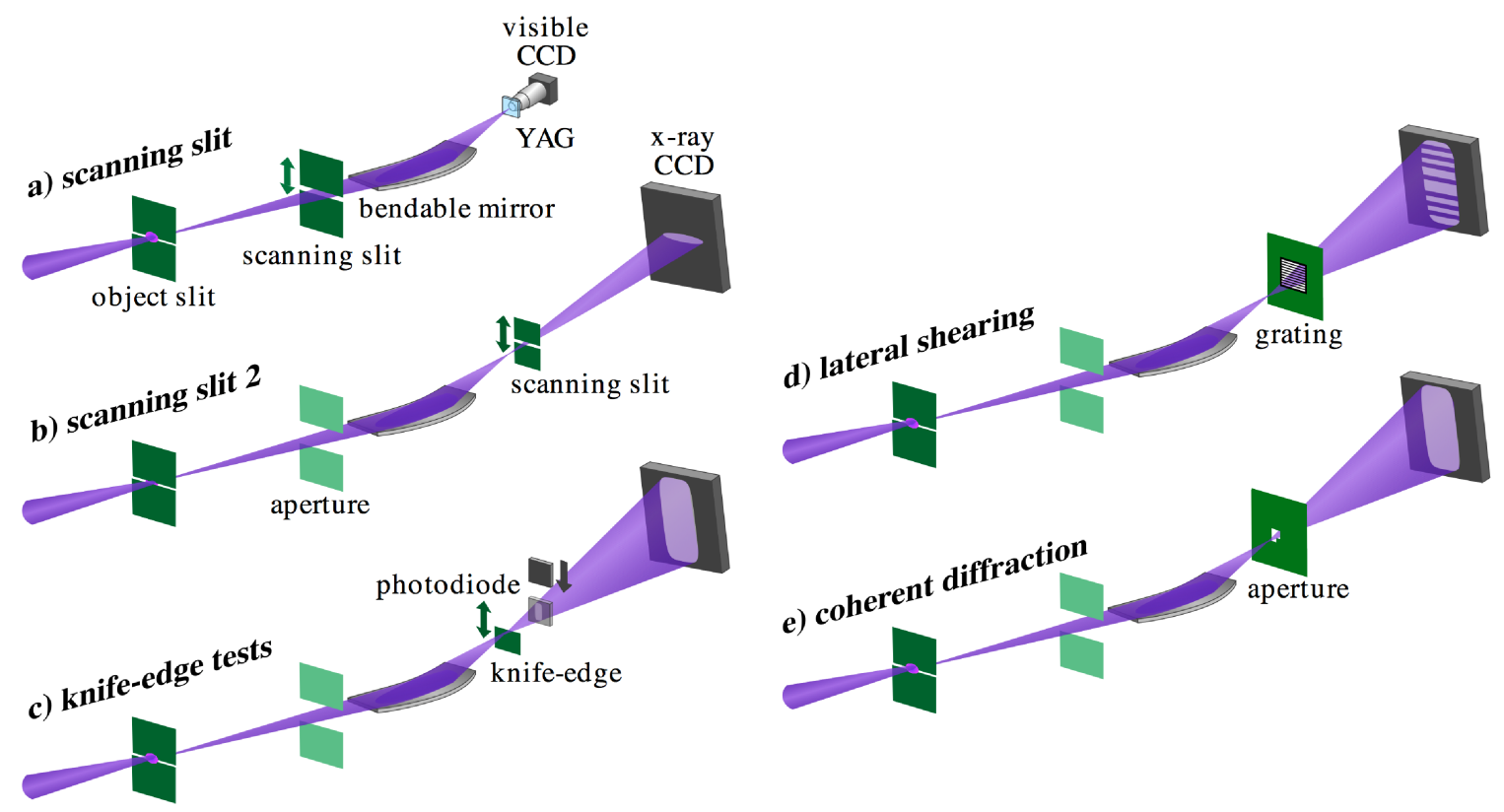

Figure 5. The in situ, at-wavelength test plan: a series of tests with increasing accuracy and sensitivity. For the purpose of demonstration and learning, a single KB mirror is used in the tests described here.

In a second test (Fig. 5c), scanning knife-edge near focus reveals the size of the focal spot, determined by all perturbations including diffraction, mirror shape error, misalignment, vibration, etc. The knife edge is moved in steps that are smaller than the diffraction-limited beam focus. In the knife-edge test described in this report, the intensity is measured with a simple photodiode placed beyond the knife edge.

\subsection{Additional tests for coherently illuminated systems}

Components for additional tests have been incorporated into the test chamber, and are the subject of ongoing measurements; briefly, these include the following.

The scanning-slit test has two additional variations that record the transmitted beam on a CCD detector placed far downstream (approximately $1.6 \mathrm{~m}$ beyond the focal plane). Compared with measurement in the focal plane, the additional detector distance enables greater precision in the slope measurements, but this technique requires calibration to achieve proper wavefront curvature for a specific focal plane of interest. With the mirror aperture fully illuminated, a small scanning-slit can be used near (but not in) the beam's focal plane (Fig. 5b), to measure the wavefront slope downstream of the mirror.

A second version of the knife-edge test (Fig. 5c) records a series of diffraction patterns using the downstream x-ray CCD camera. Such measurements enable quantitative wavefront slope measurement [17].

For in situ surface slope measurements, the Hartmann test may be considered the current state-of-the-art technique. In the Hartmann test, the test beam projects a shadow of a grid object on a distant CCD camera; and the mirror surface slope information can be extracted from the spot positions in the captured image. To guarantee mutually incoherent illumination of the various grid holes, the grid object is typically placed far from the focal plane [16]. However, 
achieving 100-nrad sensitivity is a great challenge for the Hartmann test given the finite precision with which the spot positions can be measured, the practical limits on the CCD distance set by space restrictions, the sizes of the CCD arrays, and the measurement solid angle.

For coherently illuminated systems, lateral shearing interferometry offers additional wavefront slope measurement sensitivity [18]. The geometry is closely related to the Hartmann test, but the grid (i.e. grating) is reduced in size and placed closer to focus (Fig. 5d). A relatively coarse grating period is used to maximize the overlap of the various diffraction orders in the CCD plane. The resulting shearing interferogram can be interpreted with standard techniques to reveal the wavefront slope in the direction of the shear.

Coherent diffraction is a non-traditional, emerging interferometric technique, in which the focused light interacts with a specially designed diffracting structure (Fig. 5e) and the wavefront is reconstructed using mathematical retrieval algorithms.

\section{INITIAL MIRROR ALIGNMENT}

The first steps in the mirror alignment procedure are the adjustment of the entrance-slit, followed by setting the mirror's vertical-translation and coarse tilt angle. A schematic of the key optical components of the test chamber are shown in Fig. 6 for reference.

\subsection{Optimizing the beam-defining aperture and entrance-slit}

A removable photodiode downstream of the object plane provides simple feedback for the optimization of the entranceslit and beam-defining aperture positions. These two elements are held on separate $x y$ stages. The entrance-slit array can be translated out of the beam entirely, to ensure optimal, initial alignment of the focused beam through the $200-\mu \mathrm{m}$, tungsten aperture. Once the entrance-slit position has been optimized for peak transmission the object-plan photodiode is removed. In practice, we found that the beamline mirrors or the entrance slit required occasional, small adjustments to maintain peak throughput over timescales of several hours.

\subsection{Setting the test mirror translation}

With the scanning-slits wide open, the test mirror is retracted vertically so the full beam passes unimpeded to the image plane. The first goal is to raise the mirror into the position where the illumination central ray is close to the center of the mirror's clear aperture. Owing to the glancing-incidence geometry and the mirror's curvature, the ideal position of the central ray is slightly downstream of the mirror's geometric center, where it coincides with the center of the reflected wavefront. To assess the alignment, we rely on a visible-light camera and microscope objective, which magnifies the image of the x-ray light incident on a $100-\mu \mathrm{m}$-thick yttrium aluminum garnet (YAG) crystal placed in the mirror's designed focal plane. Initially, the full width of the beam is observed; the mirror is raised until the shadow of the downstream edge of the mirror reaches the position of the central ray, as observed in the microscope image. This

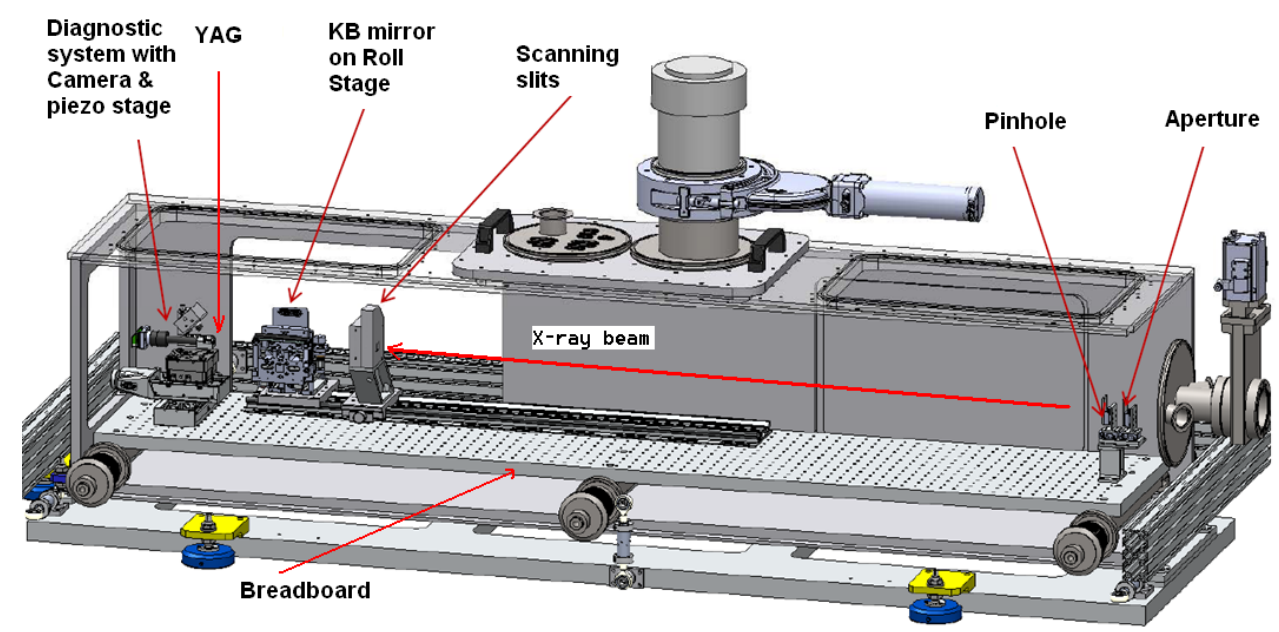

Figure 6. Cutaway drawing of the test chamber's internal components arranged for the scanning-slit test 
mirror position is recorded. At this point, half of the incident beam is reflected upward. The microscope position is then raised to observe the reflected light. The next step is easier with the YAG displaced from focus. The mirror is adjusted upward until half of the reflected beam is blocked by the upstream edge of the mirror, and this second position is recorded. The optimal mirror position is midway between these two values.

\subsection{Coarse mirror tilt adjustment}

The focal length of a curved mirror in grazing-incidence geometry is strongly dependent on the mirror's tilt angle. We use this fact to coarsely and quickly set the mirror tilt (pitch) alignment. With the YAG roughly positioned at the designed focal plane ( $\sim 120 \mathrm{~mm}$ away from the mirror center), the observed beam width can be measured as the tilt angle is adjusted (Fig. 7).

In our system, the YAG crystal microscope uses a $6 \times$ objective $(\mathrm{NA}=0.1)$, and each pixel in the recorded image corresponds to approximately $0.775 \mu \mathrm{m}$ on the surface of the crystal. Due to aberrations and visible-light blooming, the effective resolution is closer to $6 \mu \mathrm{m}$, which is insufficient for high-resolution optimization of the mirror focusing using this technique alone. Optimizing the mirror tilt in this way, we achieved a minimum measured FWHM of approximately 7 pixels, or $6 \mu \mathrm{m}$, and a clear indication of approximately where the optimized tilt angle would be found. Figure 8 shows a detail of the beam measurement with the mirror tilt at an angle close to the minimum FWHM.

Image slit Y FWHM vs. KB tilt

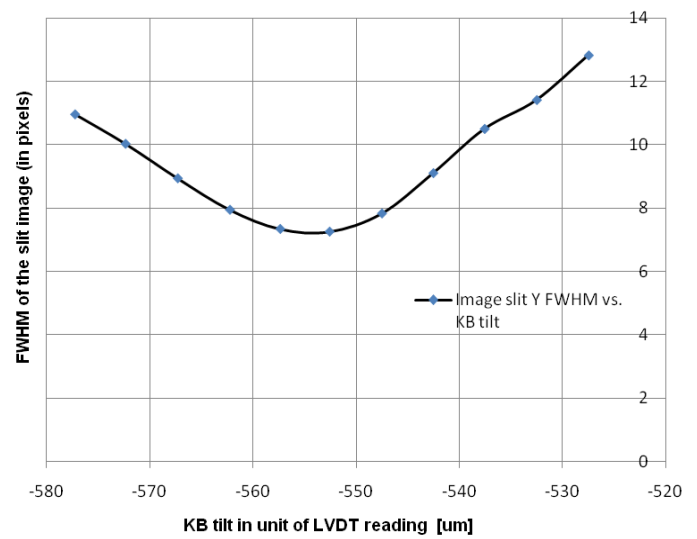

Figure 7. Dependence of the FWHM of the slit image in the $y$ (vertical) direction on the mirror pitch angle (tilt). The minimum of the measured dependence corresponds to the nominal grazing incident angle of $8 \mathrm{mrad}$. The horizontal axis of the plot is the position of the motor that controls the $\mathrm{KB}$ tilt, measured in $\mu \mathrm{m}$ of displacement; the vertical axis is the beam width (in pixels). The minimum value (approximately 7 pixels, or $6 \mu \mathrm{m}$ ) is limited by defocus and blooming in the visible-light image of the YAG crystal.

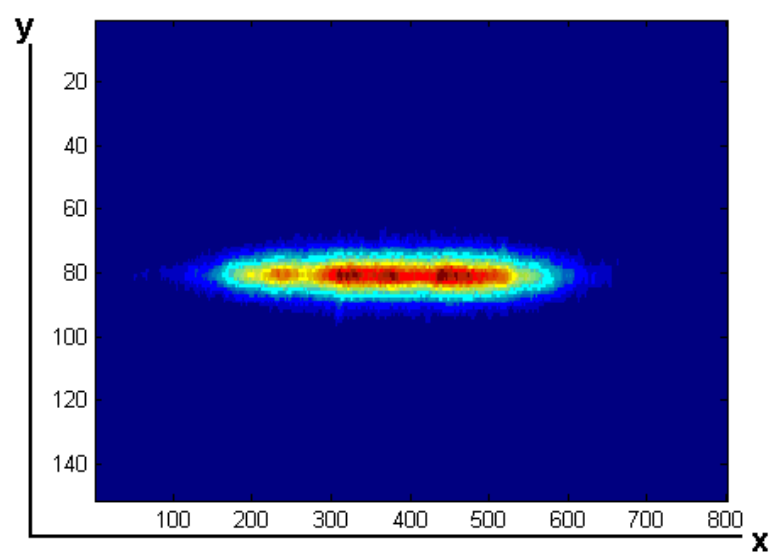

Figure 8 . The initial slit image with scanning-slits widely open. The units here are pixels. 


\subsection{Mirror initial alignment assessment with the scanning-slit}

The first in situ, at-wavelength metrology is the scanning-slit test, described in the Section 4.1. We use a motor-driven, four-jaw slit assembly produced by JJ X-Ray, placed just upstream of the test mirror (Fig. 6). The slits are narrowed vertically to $50 \mu \mathrm{m}$ width, to create a beam footprint that isolates approximately one-tenth of the mirror aperture. With the YAG crystal roughly at the designed focal plane distance, the mirror produces a visible focused line image. A series of 13 images are recorded as the slit position is scanned; the resultant beam centroid position dependence on the slit reveals the wavefront slope variation along the mirror surface.

To measure the vertical position of the beam, we average the measured intensity parallel to the line ( $x$-direction), restricting the calculation to only the brightest, central portion of the image. Before averaging, a intensity threshold is applied to eliminate noise (the threshold level is typically set to $10-20 \%$ of the peak intensity) and a median filter is applied only at the locations of any remaining isolated bright pixels.

When the beam is well focused, its vertical size is small, and it only spans a few pixels (fewer than 10) above the intensity threshold. When the threshold is low, the accuracy of the centroid calculation may be acceptable. However, centroid calculation on such a dataset could be vulnerable to errors caused by the limited number of points used in the fit and from unpredictable systematic errors induced by the threshold's ability to eliminate some points from measurement asymmetrically. We therefore recommend either careful centroid measurement with high flux (low threshold) or a polynomial fit to the beam's peak position to determine the vertical beam position. Repeatability tests show that the vertical beam position can be measured with a precision smaller than $0.08 \mu \mathrm{m}$. This corresponds to a local slopemeasurement uncertainty of approximately $0.6 \mu \mathrm{rad}$ for a ray from the mirror center.

Figure 9 shows the measured relative beam position versus the slit's step number as the slit is scanned across the incident beam during one step of the alignment procedure. The plot may be interpreted as a geometrical ray fan in the $y$ direction. Here, the ray error varies by approximately $5 \mu \mathrm{m}$, peak-to-valley, and $1.8 \mu \mathrm{m}$ RMS, which is significantly larger than the RMS geometric spot size expected from the mirror shape error measured ex situ (Fig. 3), thus indicates residual alignment error. In a simple interpretation, the finite slope reveals the presence of defocus, and the second-order curvature indicates the presence of coma. However, detailed analysis shows that when the YAG is not exactly in the mirror's focal plane, the non-linear relationship between the scanning-slit and the beam position on the mirror surface (Fig. 5a) induces an observable, non-linear motion of the measured beam position. Despite this subtlety, when the mirror is aligned and the YAG is positioned in the focal plane, the beam will remain stationary as the slit is translated. Therefore, the optimal trace is always flat.

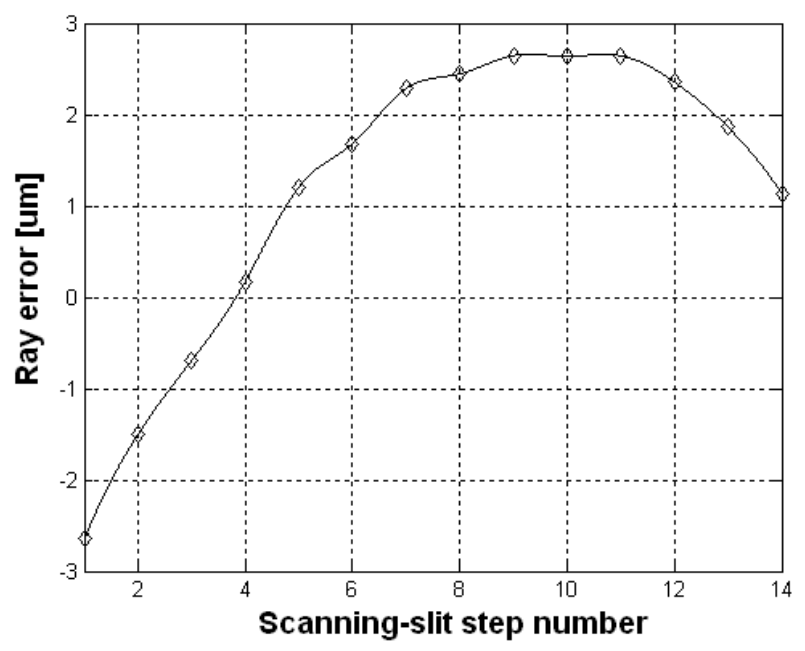

Figure 9. Measured beam position ( $y$-direction) vs. scanning-slit step number, during one step of alignment procedure.

\section{OPTIMIZING THE MIRROR ALIGNMENT}

For an optimally shaped KB mirror placed on a beamline, there are two critical first-order alignment parameters: the mirror tilt (pitch) angle and the longitudinal focal plane position. In this section we demonstrate that the method of 
characteristic functions, originally developed for the optimal setting of bendable optics $[14,19,20]$ with two free parameters, can be extended to systematically optimize the alignment of a single KB mirrors in situ.

It can be shown $[19,20]$ that due to the near linearity of the bending problem, the minimum set of data necessary for tuning two bending couples consists of three slope traces measured before and after a single adjustment of each bending couple. From the measurements, the experimental characteristic functions of the benders, which describe the response of the mirror surface shape to a unit change of the bender couples, are found. An efficient algorithm used for finding optimal bender settings is based on linear regression analysis using the measured characteristic functions of the benders [6]. The resulting approximation to the functional dependence of the desired mirror shape provides nearly final settings for the benders in one or just a few iterations.

In the case of mirror alignment, the near linearity of the problem is not as obvious. However, since the mirror is prealigned to its designed geometry and the residual alignment error may be regarded as a perturbation from the ideal geometry (Table 1), the KB mirror alignment problem should be nearly linear from a perturbation point of view. Thus, we have found that an analogous characteristic functions measurement technique is applicable to the KB mirror alignment, using the $\mathrm{KB}$ mirror tilt and the focal plane position as the two free parameters. Here we empirically demonstrate the efficiency of this alignment procedure.

\subsection{Calculating the characteristic functions}

Similar to the bender characteristic functions $[14,19,20]$, the alignment characteristic functions of a mirror placed on a beamline, $f_{p}\left(\rho_{i}\right)$ and $f_{d}\left(\rho_{i}\right)$, are determined by calculating the responses of the vertical beam position traces $y_{p, d}\left(\rho_{i}\right)$, based on unit changes in the two parameters: $\Delta p$ for the pitch angle, and $\Delta d$ for the focal plane position, respectively:

$$
f_{p}\left(\rho_{i}\right)=\frac{y_{p+\Delta p, d}\left(\rho_{i}\right)-y_{p, d}\left(\rho_{i}\right)}{\Delta p} \text {, and } f_{d}\left(\rho_{i}\right)=\frac{y_{p, d+\Delta d}\left(\rho_{i}\right)-y_{p, d}\left(\rho_{i}\right)}{\Delta d},
$$

where $\left\{\rho_{i}\right\}$ are the positions of the scanning-slit at which the beam positions $y_{p, d}\left(\rho_{i}\right), y_{p+\Delta p, d}\left(\rho_{i}\right)$, and $y_{p, d+\Delta \mathrm{d}}\left(\rho_{i}\right)$ are measured. These positions need not be uniformly spaced, so long as they are repeatable and cover the mirror's useful clear aperture.

In practice, to measure the characteristic functions, three scanning-slit measurements are performed with two different mirror tilts, $p$, and two YAG crystal positions, $d:\{(p, d),(p+\Delta p, d),(p, d+\Delta d)\}$. The characteristic functions are calculated from these three successive measurements following Eqs. 1a and $1 \mathrm{~b}$.

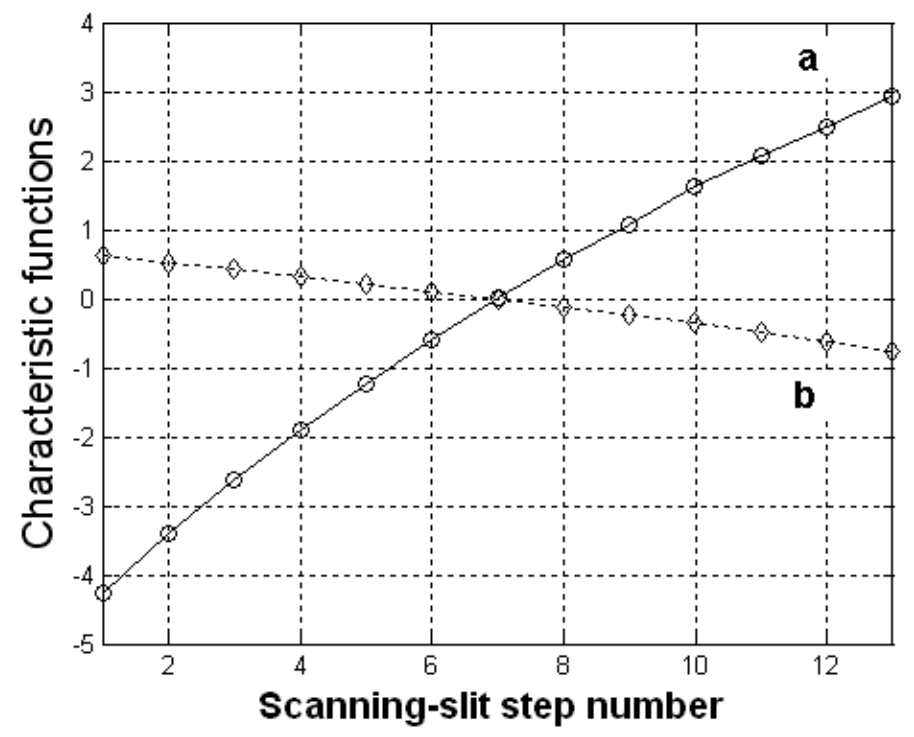

Figure 10. The measured alignment characteristic functions of the test $\mathrm{KB}$ mirror. (a) The focalplane characteristic function is shown in $\mu \mathrm{m}$ of beam displacement per $\mathrm{mm}$ of longitudinal change $(\mu \mathrm{m} / \mathrm{mm})$. (b) The mirror-pitch characteristic function is shown in $\mu \mathrm{m}$ of beam displacement per $\mu \mathrm{m}$ of linear stage motion $(\mu \mathrm{m} / \mu \mathrm{m})$.

Figure 10 shows the measured alignment characteristic functions for the test KB mirror, found in this way. The step size of the scanning-slit is $50 \mu \mathrm{m}$. Each characteristic function is dominated by a linear term corresponding to wavefront 
defocus. This confirms our expectation that both degrees of freedom have a strong influence on the measured defocus. Yet both functions also contain a smaller non-linear term, corresponding to higher-order slope errors. Optimizing the mirror alignment therefore requires a simultaneous minimization of the linear, defocus term and the higher-order, nonlinear components.

Starting from the alignment state shown in Fig. 9, we used these characteristic functions to perform the mirror alignment in two iterations, depicted in Fig. 11. The first iteration (Fig. 11a) eliminated most of the higher-order aberrations, leaving a nearly linear trace, and reducing the relative tangential RMS ray error from $1.8 \mu \mathrm{m}$ to $0.64 \mu \mathrm{m}$.

A second iteration, using the measured ray error in Fig. 11a and the same characteristic functions (Fig. 10) as input, further improved the alignment. The second iteration indicated a $0.19 \mathrm{~mm}$ YAG crystal translation, and a $20 \mu \mathrm{rad} \mathrm{KB}$ tilt-angle change (a $2-\mu \mathrm{m}$ translation of the KB tilt stage). The trace shown in Fig. 11b was recorded following the second iteration. The RMS beam position variation (ray error) was reduced to $\sim 0.1 \mu \mathrm{m}$.

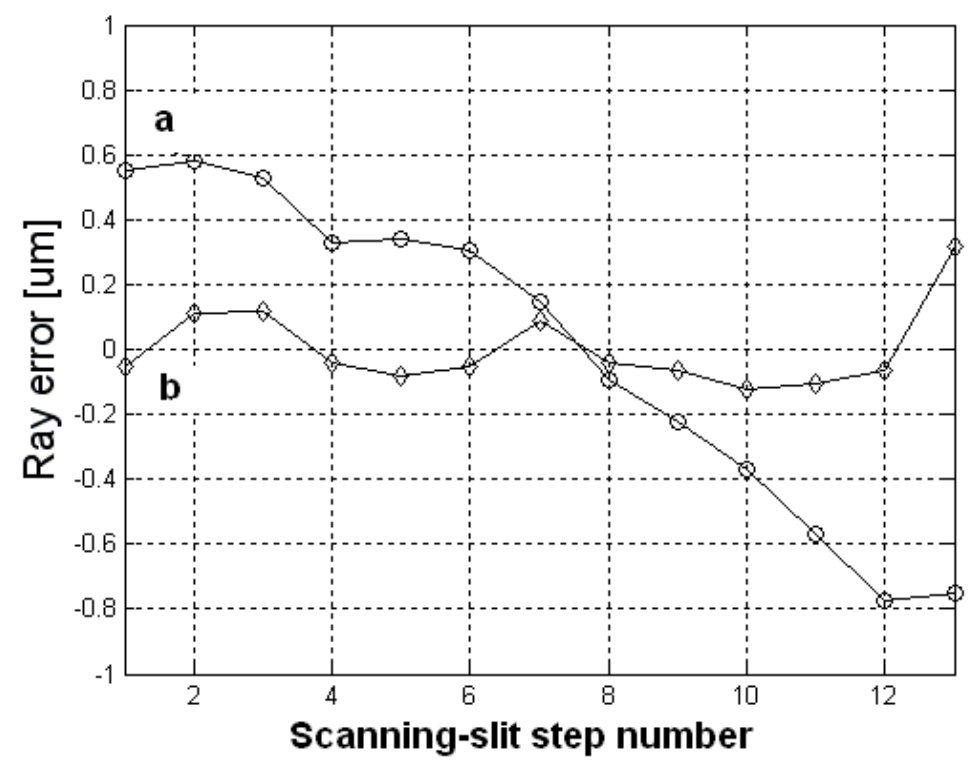

Figure 11. The focus error measured after each of two alignment iterations: (a) then (b). The relative $y$-centroid position of the slit image measured on the YAG is shown for each position of the scanningslit.

The alignment method using characteristic functions was performed repeatedly from different initial alignment positions with similar results. We observed that in as few as two iterations, the alignment converges to the settings with geometrical ray error on the order of $100 \mathrm{~nm}$ RMS, consistent with the RMS geometric spot size expected from the mirror shape error measured ex situ (Fig. 3). The focusing accuracy of the final setting is limited by inherent aberrations of the mirror shape. While noise in the measured characteristic functions can limit the alignment accuracy, its significance is reduced as the algorithm converges toward the optimal settings and the alignment step sizes naturally decrease. Noise in the characteristic function measurements can be reduced by selecting step sizes, $\Delta p$ and $\Delta d$, that are large enough to induce beam positions changes that are substantially greater than the beam-position measurement uncertainty. In the alignment steps, we found that the repeatability of the stage motions under micron-scale actuation, can also limit the accuracy of the alignment.

\section{KNIFE-EDGE SPOT SIZE MEASUREMENTS}

The scanning-slit test described above relies on the measurement of the beam peak or centroid position, and thus measures the geometrical ray error across the mirror's clear aperture. Beam size estimation from these measurements do not reflect the physical effects of diffraction. Furthermore, using a several-second CCD integration time for each image, the test is relatively insensitive to vibrations that may blur the focal spot.

To measure the actual, physical focal spot size, we insert a single-element photodiode downstream of the focal plane, and scan a nano-fabricated knife edge across the beam's focal point. The knife edge was created with electron-beam lithography as a $200-\mu \mathrm{m}$ wide horizontal slit (hundreds of times larger than the beam width in focus) made from a 
1- $\mu$ m-thick gold absorber on a 100 -nm-thick silicon-nitride membrane. The knife is translated in small steps using a translation stage capable of $42 \mathrm{~nm}$ resolution. As the edge scans through the beam, the photodiode current reveals the sharpness of the focus (Fig. 12a). The derivative of the current reading (Fig. 12b) provides a measurement of the spot's width.

As shown in Fig. 12, the knife edge scan step size was $50 \mathrm{~nm}$. By linear interpolation of the photocurrent's derivative (Fig. 12b), we estimate that the FWHM of the focal spot size is approximately $120 \mathrm{~nm}$. The beam has a noticeable tail on one side of focus, which indicates possible residual coma in the mirror surface figure together with possible scattering on mirror surface, and will be investigated in future tests with improved sensitivity and accuracy (Fig. 5).
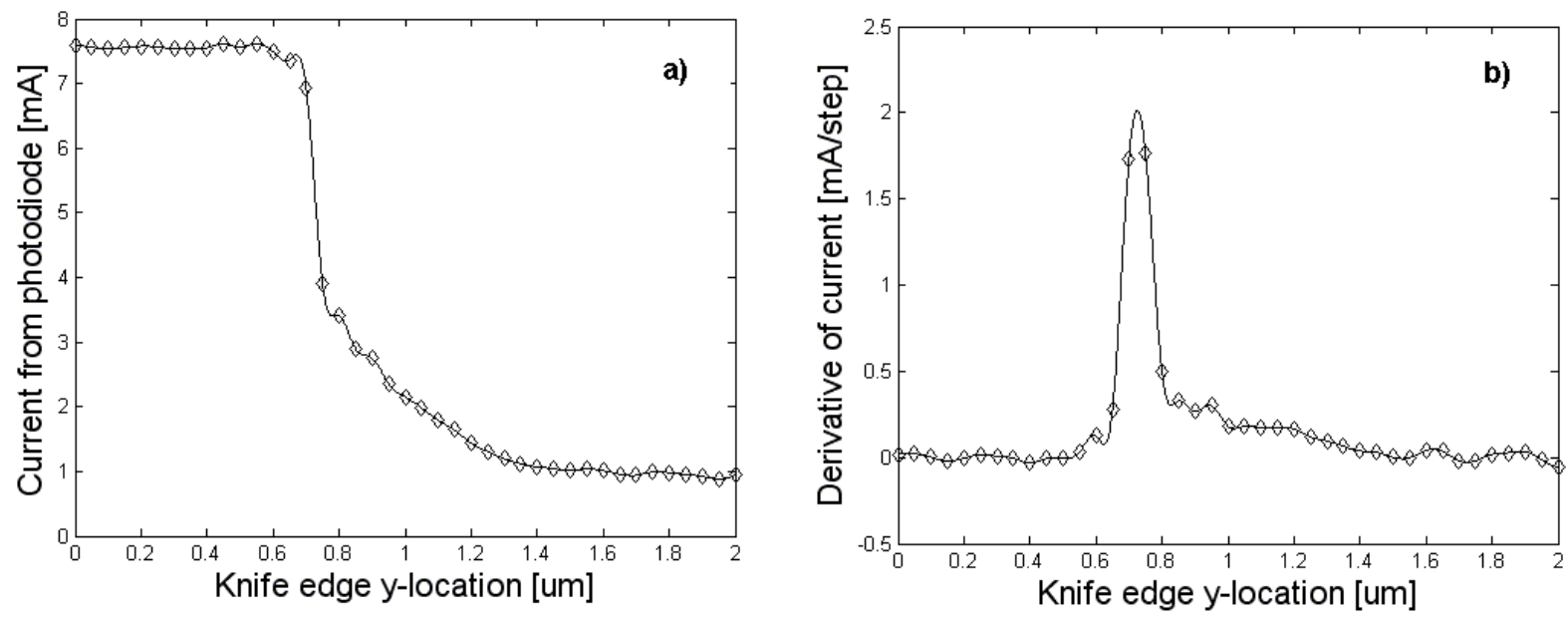

Figure 12. Focal spot size measured with a photodiode and a scanning knife-edge in the focal plane. The knife edge step size is $50 \mathrm{~nm}$. (a) The measured photocurrent shows an abrupt drop as the knife crosses through the beam. (b) The derivative of the photocurrent enables an estimation of the focal spot FWHM size: $\sim 120 \mathrm{~nm}$.

\section{CONCLUSIONS AND FUTURE WORK}

The goals of nano-focusing x-ray optics motivate the development of tests with sub-100-nm focal spot and sub-100-nrad slope error sensitivities. Soft x-ray at-wavelength metrology development at the ALS is proceeding hand-in-hand with ex situ visible-light mirror metrology to create an effective, transferable testing methodology that can be used to improve beamline performance with minimal hardware requirements.

We report the current status of at-wavelength metrology development at the ALS. A single, elliptically-bent, KB mirror element serves as a test mirror for our investigations. Following ex situ bending optimization at the ALS OML, the temperature-stabilized mirror was installed in a dedicated vibration isolated metrology endstation, and subjected to repeated in situ alignment and tuning characterization.

In this paper, we primarily concentrated on optimizing the KB mirror alignment to achieve the smallest possible spot size. Coarse alignment steps were performed to pre-align the mirror's vertical displacement and tilt angle. Fine alignment was conducted to optimize the KB mirror tilt angle and focal plane positions with a scanning-slit technique that uses the method of characteristic functions and linear regression, adapted from the mirror-bending methodology.

Using these procedures, we demonstrated a fine alignment state with a measured ray-error magnitude of $\sim 400 \mathrm{~nm}$ peakto-valleys, and $\sim 100 \mathrm{~nm}$ RMS. Independent verification of the spot size was performed with a knife edge test that showed a FWHM value of $\sim 120 \mathrm{~nm}$.

At this fine setting, the mirror is aligned well enough for the more advanced tests (described in Section 4) to perform the in situ mirror fine tuning and to surpass 100-nrad slope measurement accuracy, as will be described in future work. 


\section{ACKNOWLEDGEMENTS}

The Advanced Light Source is supported by the Director, Office of Science, Office of Basic Energy Sciences, Materials Sciences Division, of the U.S. Department of Energy under Contract No. DE-AC02-05CH11231 at Lawrence Berkeley National Laboratory.

\section{DISCLAIMER}

Certain commercial equipment, instruments, or materials are identified in this document. Such identification does not imply recommendation or endorsement by the US Department of Energy, LBL, LBNL, ALS, or BNL, nor does it imply that the products identified are necessarily the best available for the purpose.

\section{REFERENCES}

[1] L. Assoufid, O. Hignette, M. Howells, S. Irick, H. Lammert, P. Takacs, "Future metrology needs for synchrotron radiation grazing-incidence optics," Nucl. Instrum. and Meth. A 467-468, 267-70 (2001).

[2] F. Siewert, H. Lammert, T. Zeschke, "The Nanometer Optical Component Measuring Machine," in: A Erko, M. Idir, T. Krist, A. G. Michette (Eds), Modern Developments in X-Ray and Neutron Optics, Springer, Berlin 2008.

[3] J. L. Kirschman, E. E. Domning, W. R. McKinney, G. Y. Morrison, B. V. Smith, and V. V. Yashchuk, "Performance of the upgraded LTP-II at the ALS Optical Metrology Laboratory," Proc. SPIE 7077, 70770A-1-12 (2008).

[4] V. V. Yashchuk, S. Barber, E. E. Domning, J. L. Kirschman, G. Y. Morrison, B. V. Smith, F. Siewert, T. Zeschke, R. Geckeler, A. Just, "Sub-microradian Surface Slope Metrology with the ALS Developmental Long Trace Profiler," Nucl. Instr. and Meth. A 616, 212-23, (2010).

[5] R. D. Geckeler, "ESAD Shearing Deflectometry: Potentials for Synchrotron Beamline Metrology," Proc. SPIE, 6317, 63171H-1-13 (2006).

[6] Sheng Yuan, M. Church, R. Celestre, G. Morrison, W. McKinney, T. Warwick, K. Goldberg, V. Yashchuk, "Surface slope metrology and interferometric wave front measurements on deformable soft x-ray mirrors performed in the laboratory and in-situ at-wavelength," The Tenth International Conference on Synchrotron Radiation Instrumentation (Melbourne, Victoria, Australia. September 27-October 2, 2009)

[7] S. Mourikis, W. Jark, E. E. Koch, V. Saile, "Surface temperature and distortion of optical elements exposed to high power synchrotron radiation beams," Rev. Sci. Instrum. 60 (7), 1474-8 (1989).

[8] T. Warwick, S. Sharma, "Thermal effects and mirror surface figure requirements for a diagnostic beamline at the Advanced Light Source," Nucl. Instr. and Meth. A 319, 185-7 (1992).

[9] N. Kihara, K. Mashima, S. Miura, A. Miyaji, K. Wakamiya, S. Ichikawa, "Thermal and deformation analyses of side-cooled monochromator mirrors for the Spring-8/Figure-8 soft x-ray undulator," J. Synchrotron Rad. 5, 811-3 (1998).

[10] K. Freund, "Challenges for synchrotron x-ray optics," Proc. SPIE 4782, 1-12 (2002).

[11] Sheng Yuan, M. Church, V. V. Yashchuk, K. A. Goldberg, R. S. Celestre, W. R. McKinney, J. Kirschman, G. Morrison, T. Noll, T. Warwick, H. A. Padmore, "Elliptically Bent X-Ray Mirrors with Active Temperature Stabilization," X-Ray Optics and Instrumentation, in press.

[12] Sheng Yuan, K. Goldberg, V. V. Yashchuk, R. Celestre, T. Warwick, W. R. McKinney, G. Morrison, S. B. Rekawa, I. Mochi, H. A. Padmore, "At-wavelength and optical metrology of bendable x-ray optics for nanofocusing at the ALS," Extended Abstract in the Digest of the OSA Optics and Photonics Congress: Frontiers in Optics 2009, Laser Science XXV, Special Symposium on Optics for Imaging at the Nanoscale and Beyond (San Jose, California, USA, October 11-15, 2009).

[13] M. Kunz, N. Tamura, K. Chen, A. A. MacDowel, R. S. Celestre, M. M. Church, S. Fakra, E. E. Domning, J. M. Glossinger, J. Kirschman, G. Y. Morrison, D. W. Plate, B. V. Smith, T. Warwick, V. V. Yashchuk, H. A. Padmore, and E. Ustundag, "A dedicated superbend x-ray microdiffraction beamline for materials-, geo- and environmental sciences at the Advanced Light Source,” Rev. Sci. Instrum. 80 (8), 035108/1-10 (2009).

[14] O. Hignette, A. Freund, E. Chinchio, "Incoherent x-ray mirror surface metrology," Proc. SPIE 3152, 188-99 (1997). 
[15] Patrick P. Naulleau, Phil Batson, Paul Denham, David Richardson and James Underwood, "An in situ scanningslit alignment system for Kirkpatrick-Baez optics,” Opt. Comm. 212 (2002) 225-33.

[16] P. Mercère, P. Zeitoun, M. Idir, S. Le Pape, D. Douillet, X. Levecq, G. Dovillaire, S. Bucourt, K. A. Goldberg, P. P. Naulleau, and S. Rekawa, "Hartmann wave-front measurement at $13.4 \mathrm{~nm}$ with $\lambda_{\mathrm{EUV}} / 120$ accuracy," Opt. Lett. 28 (17), 1534-36 (2003)

[17] H. B. Cheng, Y. Yam, Senior member, IEEE, and H. Tong, "A Quantitative Knife-edge Testing Method for Local Deformation Evaluation in Optical Aspheric Fabrication," Proc. of the 3rd Annual IEEE Conference on Automation Science and Engineering Scottsdale, AZ, USA, Sept 22-25, 2007, 818-22.

[18] P. P. Naulleau, K. A. Goldberg and J. Bokor, "Extreme ultraviolet carrier-frequency shearing Interferometry of a lithographic four-mirror optical system," J. Vac. Sci. \& Technol. B 18 (6), 2939-43 (2000).

[19] W. R. McKinney, S. C. Irick, J. L. Kirschman, A. A. MacDowell, T. Warwick, V. V. Yashchuk, "New Procedure for the Adjustment of Elliptically Bent Mirrors with the Long Trace profiler," Proc. SPIE 6704, 67040G (2007).

[20] W. R. McKinney, J. L. Kirschman, A. A. MacDowell, T. Warwick, V. V. Yashchuk, "Optimal tuning and calibration of bendable mirrors with slope measuring profilers," Opt. Eng. 48 (8), 083601-1-8 (2009). 